\title{
La Contemplación según Plotino
}

Cuando se leen en las Enéadas las páginas dedicadas de moda expreso y directo al estudio de la contemplación, (11 1,8) es imposible evitar una primera impresión de desconcierto. Porque se diría que, segín Plotino, todo es contemplación: la naturaleza, el alma, la razón. la inteligencia. Y así este concepto a causa de su gran extensión, parece que perdiera su especificidad. Mas esta impresión desaparece, y la meditación la reemplaza por un sentimiento más adecuado y más alto: la admiración ante la profundidad y los vastos alcances de la doctrina que es al propio tiempo una psicología, una estimativa y una ontologia de la contemplación.

Lo que anarace con inmediata claridad a través de todas las Enéadas es la suprema excelencia de la contemplación que, entendida como la forma de la existencía en que el ser reposa en sí mismo elevándose sobre toda forma de alteración y de cambio, se da como la forma más plena y alal vez Cnás bura a que puede aspirar el alma en su réación con el ser. Conilo qual, en el sámbito de la vida humana, la contemplación es superior a la acción, y en la vida universal la contemplación no es otra cosa que el ser mismo en su más augusta permanencia y plenitud. Encontramos pues una clara estimativa de la contemiplación, un sentido de veneración por este modo de la existencia y de la vida espiritual, una valoración en suma que puede servirnos de guía, de clave, de indicio para alcanzar positivamente su sentido metafísico y su proyección religiosa y mística.

Acaso el supremo resorte, la más íntima fibra psicológica de la tensión metafísica en el pensamiento de Plotino es su amor a la unidad hipostasiada en el Uno Puro. El Uno es el Bien, el Bien es el Uno. Suprema eminencia hacia la cual tienden la nostalgia y el anhelo místicos, origen y suma del amor $y$ del ser en que al par se funda y se acaba la meditación del gran alejandrino. "La inteligencia, asierta, es el primer acto del bien, el primer ser. Fuera de la inteligencia y al rededor de ella circula el alma; ésta mira en la inteligencia $\mathrm{y}$, contemplándola hasta en su intimidad, ella ve, por la inteligencia, 
al Dios Supremo" (1. 8,2). Vislumbrando así el objetivo y señaladas las zonas de la existencia que le son inferiores y dependientes, se ofrece al alma el camino del retorno' $Y$ de esta sierte se átirma y exalta, como fin en si misma y como vía para la accesión al Suprems, la contemplación.

Aunque sin duda los tratados de la primera Eréada fueron escritos por Plotino es una época tardía, en ellos podemos encontrar una a marera de introducción general al estudio de la obra y muy especia!mente de la contemplacićn, tanto porque la belleza sensibie que en esta Enéada se esiudia de modo principal es la puerta de acceso a la vida contemplativa, cuanto porque de sus péginas de desprende una viva impresión de conjunto sobre el sentido y el fin de la escensión mística. Ascensión que es en realidad un retorno, una odisea espiritual en que el alma, purifićńndose de todo lo mate:ial y sensible, sube hacia su palría de origen, situada más allá no sćlo del mundo de los sentidos sino de la propia belieza intelizible.

Siquiendo la inspiracićn platónica, pero prolongando, por así decirlo, las líneas o las direcciones de la intención especulativa y mística de los diálogos, Plotino desarrolla en la primera Enéada una doctina de lo bello en que encontramos el sentido ascencional de la estética platónica y una rnetafísica en que el puto platonismo es superado por Lina visión, o más exactarnente por una intuición filosćfica y mística más alta. El músico, asienta Plotino ponéndonos un ejemplo arquetípico, es eleva de la sifmpe percepdion lidelosonido ay la del ritmo y la figura melćcica para ascender a captar la belleza de las proporciones y de los acordes, en fin para llegar a la armonía inteligible y a la belleza misma que está colocada por encima de toda beidad particular.

Las cosas sensibles, los cuerpos ipor qué son bellos? Todos están de acuerdo en que una combinación de colores y de líneas armoniosa y simétrica, $O$ un acorde musical, o una rítrnica sucesićn de palabras - de simples sonidos son bellos. Pero ¿por qué lo son? ¿de dónde les viene la belleza? Las ocupaciones, las acciones también pueden ser bellas; la ciencia es bella Mas ¿de dónde proviene que todas estas cosas, cuerpos, ocupaciones, ciencia, sean bellas?

Aplazando la contestacićn a esta pregunta, nos damos cuenta de inmediato, de que hay una escala en la belleza. El primer escalón lo constituye la belleza sensible, simétrica, mensurable; luego vienen las bellas ocupaciones, las virtudes en que ya la simetria no es visible. 
aunque quizá exista, la inieligencia. A lo largo de esta escala vemos que el alma se adhiere y ama las bellas formas, las bellas ocupaciones, las virtudes, la inteligencia y cćmo esta ascensión es acompañada del rechazo a lo feo, a lo informe. Lo que nos hace comprender que las cosas bellas son conformes con la naturaleza del alma y que nuestro amor hacia ellas se funda en que tanto las formas bellas como nuestra alma participan en una idea.

Los cuerpos tienen una forma impresa en elles desde lo alto, y esta impresión, esta razón divina, iluminativa, los hace bellos. Esta forma, esta razón, esta idea unifica sus partes y les armoniza y combina iormando un todo. Por eso tanto més bellos son los cuerpos cuanto más próximos están a lo incorpóreo. Y aquí, dice Plotino dando expresión a la vez a una idea filosófica y a un sentimiento de elta poesía: "La belleza de un color simple le viene de una forma oue domina la oscuridad do la materia y de la presencia de una luz incorporal que es razón o idea. De donde resulta que, entre todos los cuerpos, el fuego es bello en sí mismo: entre los oiros éementos, él ocupa ramgo de idea; es el más elevado por su posición, y el más ligero de todos los cuerpos, porque es vecino de lo incorporal; es solo y no acoje en sí los otros elementos, mientras que los otros lo acojen en sí; puesto que e!los pueden calentarse mientras que él no se puede enfriar. El alumbra y brilla porque es una idea" (1. 6,3).

Al pasar de laibelleza sensible a las bellezas más elevadas hay en la dostrina de Plotino alga digno de notarse porque nos revela con claridad la intención espiriticil de su metaísica estética. La belleza sensible es amable puesto que viene de la idea, pero deke ser superada por formas más puras y nobles de contomplación. Subida cuyo elierito, cuya atracción y cuya flama están consituídas por el amor, que en cada grado de la vida del alma la lleva a una aliura cada vez más espiritual, más diáfana $y$, para emplear una palabra que encierre el sentido de la vocación estética, inteelctual y mística que inspira toda la especulación de Plotino, cada vez más divina.

En el camino de la contemplación, la belleza sensible nos invita a entrar en otra esfeta, suprasersible, aunque inmediatamente unida a la sensibilidad: la esfera de las relaciones, proporciones, figuras. Pero el alma no debe detenerse en este nivel sino que dẹce alzarse a la belleza de otros objetcs superiores: las bellas ocupaciones, las artes. las virtudes, las ciencias. Y esa elevación será realizada por obra del amor guiado por la dialéctica, ciencia admirable que define los obje- 
tos, los clasifica, determina, en cada caso si la existencia les pertenece o no, que, en fin, no sólo posee los teoremas sino las realidades mismas. "Técnica trascendente" (1) Llamará el profesor Gercía Baca a esta dialéctica, que nos hace remontar la condición de creaturas, hacia la suprema soledad, firmeza y permanencia de lo absoluto.

La belleza es, cada vez más, purificación, elevación y luz secuín esta descriptiva de los pasos del alma. "Siguiendo un viejo discurso, dice Plotino, la temperancia, el coraje, toda virtud y la prudencia misma son puriifcaciones" (1. 6,6), formas, diríamos nosotros, de liberación de lo sensible, mejor aún del cuerpo; $y$ en el mismo logos agrega el filćsofo: "El alma una vez purificada se hace forma, razón; ella se vuelve incorporal, intelectual; ella pertenece toda entera a lo divino donde está la ficente de la belleza y de donde vienen todas las cosas del mismo género" (1. 6,6). La belleza intelectual es así una belleza superior porgue participa de lo divino, y además porque se da en el alma misma, "realmente aislada" de toda exterioridad, y porque, siendo contemplación de la inteligencia, en ella se identifica el alma con el ser real.

De esta suerte según la doctrina dialéctica de Plotino, cada uno de los grados en la cortemplación de lo bello recibe su valor y su sigrificacićn de una luz, de una instancia más alta que todas. Son graclos de iluminación, de perticipación en una luz eminente y que van desde la belleza de los cuerpos bellos hasta la inteligencia donde reside lá belleza de las ideas lab cual tsincembargo, con ser tan alta, no es el fin absolito, supremo; todo vez que más allá do lo bello está el Bien que es el principio $y$ el fin de toda cortemplación. $Y$ el Bien es e! Uno.

El Bien está por encima de la belleza intelectual. Pero es bello con una belleza incomparable cuya contemplación es la suprema felicidad. "Es preciso pues, leemos (1. 6,7) remontar aún hacia el Bien, hacia quien tienden todas las almas. Si se le ha visto se sabe lo que yo quiero decir y en qué sentido él es bello. Como Bien, es deseado Y el deseo tiende hacia él; pero sólo lo obtienen aquellos que suben hacia la región superior, se vuelven a él y se despojan de los ropajes que han rvestido en su descenso, como aquellos que suberi hacia los santuarios de los templos deben purificarse, dejar sus antiguas vestiduras' y subir desvestidos; hasta que habiendo abandonado, en iesta subida, todo lo que era extraño a Díos se vea solo a solo, en su aislamiento, su

(1) Introducción general a las Enésdas, Buenos Aires 1948, p. 58. 
simplicidad y su pureza, a Aquél del que todo depende, hacia quien todo mira, por quien es el Ser, la vida y el pensamiento; puesto que él es causa de la vida, de la inteligencia y el ser". Al leer esta página recordamos la desnudez de que habla San Juan de la Cruz. Y al ver más adelante como ésta unión está llena de asombro acompañado de placer, y que el alma que ama esta belleza inefable está llena de espanto, pensamos en las admirables-intuiciones de Rudolf Otto sobre "Lo Santo" y vislumbramos el profundísimo sentido no solo metafísico sino religioso de esta mística.

Para alcanzar esta visión, o mejor esta unión tan alta que trascienae toda belleza sensible es necesario huir de toda forma inferior de delectación y buscar, como Ulises, nuestra verdadera patria. "Como Ulises que escapó, según dicen, de la mágica Cirse y de Calipso, es decir que no consintió en quedarse cerca de ellas, a pesar de los placeres de los ojos y de todas las bellezas sensibles que allí encontraba. Nuestra patria es el lugar dei que venimos, y nuestro padre está allá. ¿Qué son pues este viaje y esta huída? No es con nuestros pies como podemos llevarla a cabo, puesto que nuestros pasos nos llevan siempre de una tierra a otra; no es preciso tampoco preparar un at $ə l a j e$ ri ningún navío, sino que es necesario cesar de mirar y, cerrando los ojos, cambiar esta imanera de ver por otra, y despertar esta faculiad que todo el mundo posee pero de la que pocos hacen uso". $(1,6,8$.$) . Y$ esto ¿cómo lográlo? rgCerrancónos lojoslaVlo" exterior y ejercitando "el ojo interior" que vé en lo íntimo del alma misma, consumando la unión consigo misma. Estado cuya realidad, intimidad y alteza expresa el maestro con pälabras de iluminado cuando dice: (1. 6,9) "Entonces serás una visión; ten confianza en tí; aun permaneciendo aquí, has subido; no tienen ya necesidad de guía; fija tu mirada y vé".

Y aquí el principio de semejanza viene a ser un principio de identificación. Para contemplar el sol el alma debe ser semejante al sol. Para contemplar lo bello el alma debe, ser bella. Para contemplar a Dios el alma debe ser divina. Ideas que anticipan y que quizá inspiraron el pensamiento goethiano traducido en los famosos versos:

Wär nicht das Auge sonnenhaft,

Die Sonne könnt'es nie erblicken;

Läg'nicht in uns des Gottes eigne Kraft,

Wie kc̈nnt'uns Göttliches entzücken?" 
En síntesis la purificación, la elevación y la iluminación constituirían los tres momentos ideales que se adunarían en la realidad ontológica y anímica de la coniemplación, estado en el cual el alma trasciende de la semejanza y la imagen a la identidad del Ser y del Uno.

$Y$ ahora con esta idea de conjunto, y partiendo de la semioscuridod de la naturaleze, sigamos a Plotino en el itinerario de su ascención hacia la plena luz. Y hagámoslo teniendo en cuenta que el pensamiento del filósofo es, en gran medida, cíclico, es decir, que en cada paso de su meditación repite los temas ya tocados, aunque los repite inccrporándolos, dialécticamente, en una nueva y más alta esfera de periección y de ser. Plotinc establece que la naturaleza, primer peldaño de la escala ascendente de los seres, - entendida como razón se. minâl, como forma que se imprime en la materia - es contemplación. Es contemplación, asienta, porque siendo forma, no sale de sí, ni cambia, ni se mueve como la materia con lo cual la inmovilidad se nos aparece como la primera condición esencial de la contemplación. 'Mas єn esta inmovilidad hay algo cuya consideración puede elevarnos por encima de la mera naturaleza; y consiste en que, en tanto que razón, la contemplación es al propi’s tiempo, contemplación y producción. ¿Cómo?

Hay algo de enigmático ỳ dée encomprensible aa primera vista, en estas páginas de Plotino; pero de ellas deriva una como prespectiva infinita y a través de sus fó:mulas se divisa una extraordinaria profundidad, cuando se las ha meditado intensamente. La naturaleza es una razón, una forma, un logos, que viene de una razón superior, que al propio tiempo la produce y contempla. La naturaleza es pues producida como objeto de contemplacićn y contemplada en el propio acto que la engendra, al modo como el gećmetra produce las figuras geométricas por el mero hecho de contemplarlas. En tanto que razón la naturaleza es pues objeto de contemplación, el ser de la contemplación y la contemplación misma. "Para la naturaleza ser lo que ella es, es producir; ahora bien, ella es contemplación y objeto de contemplación, puesto que ella es razón; pues, porque ella es contemplación, objeto de contemplación y razón; y por ese motivo solo ella produce". (11 I, 8,3). Con lo cual, al identificar contemplación y producción, Plotino orienta nuestro pensamiento hacia la profunda esencia de la contemplación tal como él la concibe; porque si contemplar es producir, contemplar es ser, en íntima comunión de vida, de actividad y de sustancia, el propio objeto de la contemplación. 
Mirando hacía lo alto veremos cómo la naturaleza procede del alna: vemos que ella es una alma que procede de otra alma anterior y superior. Pero el aima superior es ella misma contemplación. De esta suerte, Poitino considera, una alma que habita en ol alto y una alma que, por decirlo así, dessiende. El ailma superior habita en lo inteliGibie; por lo tanto es contemplación, aunque de una especie más alta, puesto que mientras la contemplación de la naturaleza es silenciosa y un poco vaga, la contemplación del alma se da, en lo alto, en una plen:tud y una iluminación eternas.

Precisando la dirección de su pensamiento que coincide con la gradación ascendente de to real dice Plotino: "La contemplación es un progreso de la naturaleza al alma y del alma a la intleigencia; ella se une cada vez más íntimamente con el ser que contempla; en el alma sabia ya los obietos conocidos vienen a ser idénticos al sujeto que concee porque ella aspira a la inteligencia dorde sujeto y objeto no son siro unc" (1) I, 8.8). Así en la inteiigencia la contemplación alcan$z_{\text {á }}$ su perfección mayor, su plenitud; porque en ella ya no se distingue $\in 1$ sujeto que contempia del objeto contemplado y porque en ella, 0 sea en osto nivel ontolócrico. "ser y pensar son la misma cosa". Contemplacićn viviente porque en ella el objeto no es distinto del sujeto y porque asi ella posee en su plena manifestación todo lo que es vida manifestada.

Hay pues según Plotino unacidentidad fundamental entre ser y consemplación. El ser es contemplación, la contemplación es ser. El ser se conlempla a sí mismo, y ese contemplación en su ser. Por manera que en el acto en cue el alma contempla se cumple una operación ontológica, una vuelta del ser a sí, una identificación que suprime la distancia entre sujeto y objeto y que, de este modo, en el propio objeto de la contemplación pone el ser del contemplador. "Sócrates que contempla no sobe si es una inteligencia o una alma". "Toda nuestra actividad está dirigida hacia el cbjeto contemplado. Nosotros nos convertimos en objeto. No somos ya nosotros mismos mas que en potencia". (IV. 4,2).

Mas la inteligencia por alta que sea supone alteridad entre objeto $\mathrm{y}$ sujeto, entre idea e idea, y as'i supone también un principio superior, absoluto en que la unidad perfecta $y$ pura a que aspira la vida intelectual, se realice. Principio supremo, indefinible pero evidente; se diría exigido, reclamado por la propia alteridad de la inteligencia que no podría darse tal como existe sin una unidad que la sustente sin 
dividirse ella misma. Se diría, introduciendo aquí ura calegoría que ya participa de la experiencia emocional, que esa unidad, ese Uno, es inefable y por consicruiente indefinible dentro de las categorías del pensamiento conceptual.

Pero este Primero ¿puede ser objeto de contemplación? 0 mejor ¿es en sí mismo contemplación, teoría? Parece que así como está más allá del ser -que es contemplación- deba encontrarse tambión más allá de ésta en la ezfera doncie desaparecen toda distinción y todo límite.

Mas quizá debamos deienernos en la consideración de ciertas nociones, en la mediałación de ciertas fases especulätivas, en algunos plaros de la ascensión en que Plotino nos conduce. Con ello perfeccionaremos nuestros atisbos sórre el contenicio de la contemplación, sobre todo er cuanto se reliere a la mediación ciel alma entre lo sensible y lo inteligible y a su sifuación metafísica, en que habiendo descendido, asciende $y$ en que al propio tiempo ea que se trasciende $y$ encumbra, se encuentra así misma al fínal luminoso de esta ascensión.

Como ya hemos visto, en la contemplación de lo inteligible. o sea en la contemplación propiamente dicha, asciende el alma mediante una purificación consistente en su liberación de toda propensión sensible, de todo pasión desordenada y aun de todo restro de temporalidad; más aun de iodo recuerdo. A si purfificada en si misma el alma ya no atiende a lo efímero sind arbetencily ho he lo pasajero sino lo permanente; recogida en sí misma, vive en el mundo de lo inteligible $\mathrm{y}$, al contemplarlo, se contempla a sí misma como que ella es, en su parto superior y divina, ese mismo mundo inteligible. Con lo cual la contemplación es una reintegración del alma en su propia patria, la cual, en un profundo sentido metafísico, no viene a ser sino una región superior del alma misma. Esia ascensión es un recogimiento, puesto que volviéndose a sí misma el alma se eleva a lo que elia misma es, do tal suerte que en lo que podríamos llamar el acto de la contemplación o el proceso de la contemplación se dan dos movimientos o mejor dos operaciones en un sólo acto indivisible; dos movimientos que son uno y el mismo: el recogimiento y al ascensión.

En suma, la contemplación es una forma de vida en que el alma ve lo inteligible pero no como algo extraño a ella misma sino como algo en que el aima se reconoce y se posee. En otros términos, en la contemplación el alma es o se hace inteligencia, o como dice Plotino 
condensando su pensamiento: en la contemplación el alma no simplemente vé sino que es' realmente visión.

Plotino habla a veces del alma como idéntica a la inteligencia, a lo menos en la región superior del alma. Otras llama al alma imagen de lo inteligible, verbo de la inteligencia, con lo cual el alma se distingue de la inteligencia como que es una nueva hipóstasis. El alma procede de la inteligencia. Es menos eminente y aunque divina es, por su filiación, inferior. Por lo mismo el alma vuelve a sí misma (recogimiento) y, en cierto modo, dada,su situación en la jerarquía de lo real se trasciende a sí misma en el acto de la contemplación. Aquí el alma es ella misma en cuanto sujeto contemplador y es inteligencia en cuanto objeto inteligible. Así la distinción de dos regiones del alma, superior e inferior, que no destruye su unidad, aclara en cierta medida la aparente contradicción entre el movimiento que podríamos llamar de ensimismamiento y el movimiento de trascendencia o elevación que constituye en su unidad metafísica y psicológica la contemplación.

Las Enéadas contienen numerosos y extensos pasajes psicológicos: sobre la sensación. sobre la pasión, sobre la inteligencia. Pero Plotino no siempre disocia sus consideraciones de orden ontológico de sus puntos de vista sobre los modos y formas del acontecer anímico. De modo que cuando habla de contemplación, más que en describir una experiencia interna con sus inherencias afectivas, se ocupa en definir metafísicamente la sifuación y da significación ontológica del alma. Por la contemplación el alma asume una cierta calidad, una cierta situación ontológica: asciende al par que se recoge como si el recogimiento fuese una ascensión y un retorno; y en este mismo acto el alma es visión y es inteligible. Por todo lo cual se diría que ver y ser se identifican en la contemplación.

Como condición general inherente a la actividad contemplativa, como fondo afectivo de la contemplación entendida no sólo como un estado sino como una forma permanente y transfiguradora de toda vida espiritual orientada hacia lo divino, Plotino considera la felicidad, goce tranquilo que llena el alma del sabio, elevada por sobre lo terreno y transitorio a la visión de lo eterno. Ni la enfermedad ni la muerte turbarán la serenidad de esta vida que vive más allá del tiempo con la mirada vuelta hacia la altura. Todo lo cual empero se refiere principalmente a la contemplación intelectual', toda vez que el éxtasis místico propiamente dicho no podría ser caracterizado por la mera serenidad propia de la visión intelectiva. Otras categorías del sentimiento 
serían aplicablss al éxtasis; el estupor gozoso, el espanto, el asombro. En sintesis, la aspiración mística del alma corresponde paso a paso a los grados en que se disponen los planos de lo real y los sentimientos y afectos que se dan a lo largo de esa ascensión están impregnados de honda religiosidad, como que Plotino compara el ámbito de la contemplación a un santuario en cuyo fondo mora, misterioso, el Primero, que es a la vez el fin divino del viaje espiritual.

Mas la religiosidad de Poltino apenas si es discernible de su sentido de la belleza entendida como reflejo, destello, indicio de la luz primordial, o mejor, como rayo que, a la vez, es camino por donde el alma puede volar al sol. Según hemos visto, la belleza sensible es una invilación a subir; siendo la belleza inteligible una elevada altura en sí misma y un paso hacia lo bello sín nombre ni forma. "La realidad primera, el Primera es sin forma". (VI. 7,33). "Su belleza es de otra especie que la belleza; es lina belleza por encima de la belleza". (VI. 7,32).

Aunque esa belleza suprema se comurique en cierto modo con los okjetos bellos, sensibles o inteligibles, y aunque la ascensión estética de la contemplación va de las formas visibles a las formas invisibles del reino inteligible, la belleza en sí no está encerrada en ninsuna de esas formas. Estas, limitadas como son, no pueden contener la realidad primera y total sino unicanehte reftejarlec. Las formas son bellas pero no son la belleza; Fretlejoseitnámenes lo son inferiores; Son grados oue ascieder, aunque deberán abolirse y olvidarse en el último inexpresable transporte de la cumbre. Esta belleza sin forma, principio $\mathrm{y}$ origen de toda belleza es objeto del amoroso deseo que inflama el alma y que la lleva, por el camino de la absoluta pureza y desasimiento, a ver sola al Solo.

Como indicio del reflejo afectivo de este trasporte tenemos la expresión "estupor gozoso" que denota un no saber acompañado de gozc. Es una presencia que no es la presencia de otra cosa; que no está frente al contemplador sino que se identifica y se confunde con él en un acto de amor. El amante (la inteligencia, el alma) se pierde en ei amado, $y$ en este estado todo es evidencia, verdad, gozo, luz, sin que ringuna palabra lo pueda traducir porque en él toda distinción se borra y toda determinación desaparece. Indeterminación del objeto, indeterminación del sujeto dice Brehier para caracterizar la suprema unión a cuya realidad no puede llegar el mero pensamiento, que ninguna descripción ni explicación puede hacer patente, porque está más allá de 
toda imagen e idea. Sólo el iluminado conoce este estado en su inefable misteriosidad. Es el estado místico que, trasciende ya los lindes de la contemplación propiamente dicha, aunque en un sentido elevado podría quizá decirse que es la perfección misma, el acabamiento y la suma de la contemplación, George Mehlis habla de un "ver por un no ver" (1) aludiendo a lo indescriptible de este estado en que todo es luz $\gamma$ on que desaparece toda aprehensión distinta.

No ha sido la intención de este trabajo estudiar en su integridad la filosofía de Plotino, sino exponer en forma sumaria la doctrina del gran místico sobre la contemplación. Al realizar ese propósito nos ha prarecido descubrir que la última estructura del pensamiento metafísico de Plotino, estructura que naturalmente determina sus ideas sobre la contemplación, podría representarse por el esquema de dos movimientos cpuestos y solidarios: uno, descendente emanado de la pura unidad que, sin perderse, origina la diversidad de la inteligencia y del alma, y un movimiento ascendente, involuto, por el cual los rayos de la luz primitiva tienden, o convergen ascencionalmente, a reintegrarse en el fcco inicial. En esta dialéctica vemos como la contemplación es comparable a una irradiación que secvolviese hacia el principio, realizanasí en grados de creciente elevación yl perfecciónsla unidad divina. Y vemos que acaso el último secreto de esta metafísica y de esta mística consitiría en la inexpresable necesidad que prescribe el descendimiento de*lo divino hacia la materia y que, al propio tiempo, instituye la vocación de aquello mismo que desciende hacia la reintegración en la altura. Plotino consagra así la fé del alma en su divinidad y la esperanza en su deificación absoluta mediante la puriifcación y la contemplación.

De esta suerte se diría que la contemplación deshace y en cierto modo anula el proceso descendente que va del Uno a la inteligencia y de ésta al alma. Retornar es abolir, olvidar el éxodo. Subir es deshacer, eliminar, abandonar todo ol que sea corporeidad, temporalidad y pluralidad para llegar, por esta vía de la negación, al Supremo. Pero entonces ante este imperativo del amor místico que prescribe el deshacimiento y la separación como los medios, y el retorno como el

(1) Die Mystik, München 1927, p. 89. 
fin, surge esta pregunta acaso sin respuesta dentro de los términos estrictos de la filosofía de Plotino: ¿Qué sentido tiene esta procesión cuyos pasos deben ser abolidos, borrados en la via del regreso? $\mathrm{Si}$ el único sentido es el Uno ; qué significación tiene lo vario y para qué ial despliegue del esplendor del mundo?

Marjano Iberico.

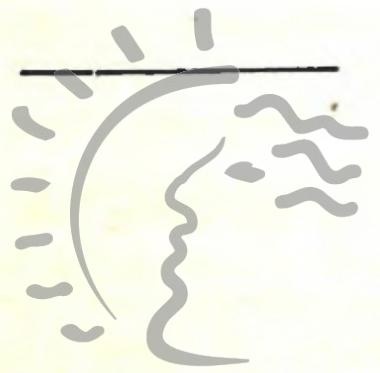

Biblioteca de Letras "Jorge Puccinelli Converso" 